interior probably large doses of the infecting bacilli, the majority of which he is capable of dealing with. Some, however, may find a nidus in the gall-bladder (as in the immunised rabbit) and there proliferate.

"The numbers of organisms that are continually discharged in the bile and resorbed through the intestinal mucosa call on the

When to this antibody-exhaustion is added the fatigues and hardships of war with its attendant diarrhoeal troubles, the chances of an ultimate systemic infection are enhanced. This view, which would attribute a systemic infection to a breakdown of immunity in a carrier presupposes some evidence of intestinal trouble preceding the attack, and in this connexion Vaughan notes that of 104 inoculated men who contracted typhoid fever 60 per cent. had antecedent diarrhœea. It was Vaughan's opinion that two of the chief causes of typhoid fever among vaccinated American troops were (1) the ingestion of overwhelming doses of the infecting organism, and (2) loss of resistance and antibodyexhaustion due to the exposures of military life. It should be mentioned that the majority of Vaughan's cases had received the saline triple vaccine and a minority the lipovaccine. In a non-vaccinated population of all ages the variety of clinical forms in which enteric disease presents itself has long been recognised. Whether in a vaccinated population there occurs a relatively greater proportion of mild cases among the attacked than would be found among the attacked of a non-vaccinated population must be determined on a basis of cultural diagnosis. The practicability of such diagnosis is not, so far as present evidence shows, enfeebled by previous inoculation.

In conclusion I may add that I have been in communication with Sir William Leishman on the question ventilated in this note. Unfortunately it may be some time yet before the relevant data can be extracted from the medical case-sheets and laboratory files of the Western front. Sir W. Leishman informs me, however, that bacteriologists in France certainly supported the views I have attributed to them in the present communication. The question is of high theoretical and practical importance, and the publication of this note may, I hope, stimulate further inquiry.

References.-1. Garrow. R. P.: THE LANCET, 1920, ii., 886. 2. WebbJohnson, A. E.: TFF LANCET, 1917, ii., 813. 3. Vaughan, V. C. : Jour Amer. Med. Assoc., 1920, pp. 1074, 1145. 4. Ledingham, J. C. G. (1920): Jour. Roy. Army Med. Corps, xxxiv., 203, 306. 5. Stone, R. L Hygiene, Plague Suppl, No. IV

\section{TREATMENT OF THE}

\section{CRIPPLING DISABILITIES OF CHILDREN}

BY G. R. GIRDLESTONE, F.R.C.S.ENG.,

STRGEON, SHROPSHIRE ORTHOPZDIC HOSPITAL; ASSTSTANT ORTHOP开DIC SURGEON, RADCLIFFE INFIRMARY, OXFORD; SORGEON, ORTHOPADIC HOSPITAL, HEADINGTON, OXFORD.

What are the main crippling disabilities?-In every list of cxipple children, whether compiled in this country, in America, or on the Continent, three main groups constitute the bulk, and do not vary much in their proportions: (A) Deformities congenital or acquired (B) paralyses; (C) tuberculosis of joint and bone. Of 1130 cases under treatment in Shropshire and North Staffordshire in 1919, and listed in Birmingham in 1911, there were: Group A, 35 per cent.; Group B, 32 per cent.; Group C, 28 per cent.; various, 5 per cent. Of 12,496 cripple children of whom the writer has been able to obtain particulars, in 35.4 per cent. the crippling disability is non-pulmonary tuberculosis, and in $52^{\circ} \tilde{5}$ per cent. it is of the A or B group. The total number to be dealt with is very large; the inquiries as to cripple children, quoted in the report of the chief medical officer of the Board of Education for 1919, give returns averaging $8 \cdot 6$ per 1000 school population, and it is clear that many of these returns are incomplete and give only a fraction of the true proportion.

Is there failure in the treatment of these aisabilities?At present the failure is exceedingly great; the principal reasons are as follows: (1) the cases do not, as a rule, get into touch with efficient treatment early enough ; (2) many of the cases nover get efficient treat ment at all; (3) a great majority of the cases suffer for lack of after-care. Every neglected congenital deformity, every "hump-back," every joint deformity due to infantile paralysis, indeed, the great majority of all cases crippled by this disease, are examples of one ox other, or even of all, of these failings.

Is the present system to blame?-There is no system ! It is the absence of any organisation, eren of any general agreement as to organisation, that makes the treatment of the child threatened with crippledom so ineffective. Some cripple children go to the union infirmary and stay there; they often have no education, and generally no treatment unless they are acutely ill, and then often only for a short period at the nearest general hospital. These are not imaginary statements ; they are based on common findings of the inquiry carried out by the Central Committee for the Care of Cripples. Some go to special hospitals for nonpulmonary tuberculosis, but, as a rule, only when they are definitely suffering from established tuberculous arthritis. To quote from a recent discussion by specialists in another sphere : "The specialist sees the difficult cases and the failures." Surely many cases of early tuberculous disease become difficult cases and lead to permanent crippledom merely because of lapse of time. That is typical of the failure of the present chaotic arrangements.

Typical Cases of Failure in Treatment.

Take an early case of tuberculous hip. A child develops a limp and cries at night. His mother knows, possibly the district nurse knows, perhaps he is even taken to the doctor. A limp and a cry or two, and for physical signs just limitation of movements of the joint in all directions, with a little flexional deformity from muscular spasm. There is no apparent sign of urgency. But actually there is no time to be lost. Prompt immobilisation of the affected joint, a bed in an open-air ward, and good feeding will prevent suffering, and will almost ensure, in perhaps nine months, full recovery, often without any permanent disability.

But only very few of these cases actually find their way into hospitals for tuberculous joints at this stage. The golden opportunity slips by, there is uncertainty, then delay, and the disease tightens its grip ! Suffering and illness increase, the text-book picture becomes easily recognisable, the joint surfaces are destroyed, there is danger to life, treatment is now not a matter of months but of years, and the child at best will be a cripple. Actual instances without number can be given of children who have paid by their prolonged suffering and illness, their joint destruction and permanent crippledom, the extreme and needless cost of our present lack of means for early ascertainment and facilities for immediate treatment.

'Take infantile paralysis. A child is feverish, perhaps very ill. One or two limbs seem powerless; the child is restless, in pain, and cries if touched. Perhaps some weeks later he is brought to a hospital and there given " massage and electricity" for a few weeks, or, if the child lives within out-patient reach, for months or years. Much recovery may occur even with this late and ineffective treatment, but the number of children needlessly crippled by preventable paralysis is very great. The profession as a whole has by no means yet realised the principle of ganglion cell rest as an urgent measure in the first stages of this disease, whether the onset of paralysis is associated with a feverish illness or not.

By the immediate immobilisation of the head, spine, and affected limbs, with correct posture ${ }^{1}$ and un. disturbed skin protection, the inflamed or poisoned cells are given the functional rest they need and cannot otherwise get. We know that even without that rest much recovery takes place (quickly or slowly); it is then reasonable to assume, as, indeed, Mackenzie's cases tend to prove, that with proper spinal and neuro. muscular rest the recovery will be quicker, more extensive, and more often complete.

1 William Mackenzie: The Treatment of Infantile Paralysis, Melbourae, 1910; Robert Jones, TrE LANCET. May 30th, 1914. 
A congenital deformity is, of course, not quite so urgent a matter, but, none the less, the ultimate result is far better and can be obtained more quickly, with less disturbance and less pain, if the defect is treated as soon as it can be detected.

Scoliosis and other deformities which develop insidiously are also at first treated easily, later with difficulty or with only partial success.

In every branch of the work early treatment tends to prevent crippledom, to forestall illness and suffering, and to eliminate the need for operation.

It has been stated that some cripples never get efficient treatment at all. How is this? It is due to lack of suitable provision for their needs, for the work cannot be done on an adequate scale by the general hospitals. The cases are slow and tedious; they need a country hospital with open-air wards, splint and appliance workshops, educational facilities, and so on. Means must be available to ensure the accurate fit and comfort of a splint, frame, plaster, or appliance, and workshops where these various needs can be supplied quickly for each case, to the exact specification and under the superintendence of the surgeon, are of the very greatest advantage. General education and handi craft training are required for these children while in hospital, for otherwise many have no teaching for very long periods, and thus have an educational as well as a physical handicap. The joint consideration and proper coördination of treatment and education is very important.

Again, in these cases there is no natural convalescence. Operations, manipulations, and even prolonged reten tion in hospital do not finish the treatment. Without thorough after-care deformities will inevitably recur, club-feet, though over-corrected, will relapse, posture, gravity, and uneven muscle-balance will cause deformity in cases of infantile paralysis, and tuberculous hips will insidiously change abduction into adduction.

To sum up, failure is due to want of (1) early treat. ment; (2) efficient treatment in suitably sited, equipped, and staffed hospitals; (3) thorough after-care.

\section{A Proposed Schenue.}

Outlines of a proposed national system to meet these needs have been worked out by the Central Committee for the Care of Cripples as follows:-

I. A central coördinating body which should work with the Ministry of Health and the Board of Education.

II. Local committees for the care of cripples in every district.

III. Hospital schools. The provision in England and Wales of an adequate number of hospital schools for crippled children with open-air wards.

IV. Each hospital school should be run under its own local committee, with its own teaching staff and handicraft workshops. Working in direct union with the hospital school should be:-

(a) Out-patient clinics for-(1) The examination of children sent up for consultation; (2) the treatment of cases which can be effectively treated as out-patients; (3) the aftertreatment and supervision of children discharged from the hospital school; (4) the maintenance of touch with old patients so that advice and help may be given as to suitable employment.

(b) Invalid schools, day and residential, for the minority of children who have not been cured or benefited sufficiently to enable them to attend ordinary classes. In large town special day schools will fill the need; but for children in other districts, who are prevented from receiving education by their persisting disability, residential schools are necessary. For the sake of simplicity and economy these should be part of the establishment of the hospital school, but in a separate wing. These children would be drafted into this when fit to leave the hospital, but would continue to benefit from the teaching, handicraft shop, and surgical supervision.

V. It is vital that those responsible for the treatment should be experienced in orthopædic work. This applies both to the surgical staff and the hospital and after-treatment sisters.

VI. There should be provision for paying patients.

VII. Wards should be available for adolescent and adult cripples and persons suffering from injuries needing orthopædic treatment.

VIII. Where efficient organisations for crippled children exist these would be welcomed in the scheme which should be sufficiently elastic to cover some diversity of method.

the Care of Cripple Children, Brit. Med.Jour., May $22 n d, 1920$.
The essential principles of the proposed scheme are those already stated, and aim at treatment, very early, very efflient, and very complete.

The suggested hospital and clinic organisation is developed from an existing unit which has been doing this work in a county area for a good many years. There the organisation was gradually developed to meet the difficulties already mentioned, the tragedy of children coming into hospital with established disease, deformity, and paralysis, and of others readmitted with relapse owing to the impossibility of keeping touch with all discharged cases, of whom many live at considerable distance.

\section{Hospitals for Cripple Children.}

The essence of the system is the establishment of hospitals for all types of cripple children to serve a more or less definite district-say, one, two, or three counties; hospitals which should accept responsibility for every cripple right through the district, organise team-work, and enlist in the team every doctor and every health worker. Success depends on a true spirit of coöperation.

Scattered orthopadic clinics.-The working of scattered orthopædic clinics is described in a leaflet used in con nexion with the organisation of clinics in another district.

After describing the work of the children's section of the hospital in the treatment of all types of crippled children this leaflet goes on to say:-

"But, if the hospital is to do its work well, it must achieve not only early treatment but also thorough after-care. Everyone is familiar with patients who have suffered from one or other of these conditions, have had a period of hospital treatment, have apparently been put right, but two years later have been just where they were before, solely as a result of deficient after-care. The very great majority of children are able to become out-patients while still in plaster-of-Paris or wearing appliances. The renewal of the former, and the upkeep, readjustment, and replacement of the latter mean constant work, and at the same time careful watch must be kept for any sign of relapse. This work must fall to the lot of the hospital staff who have carried out the active treatment, and have the enormous advantage of a workshop where any repair, alteration, or renewal of appliance or boot may be done quickly and to the exact requirements of the surgeon.

It was for this after-care, which has to be kept up for years, that the local orthopædic clinics were started in connexion with the Shropshire Orthopædic Hospital, for it was found quite impossible to bring all the old cases up to the hospital frequently. Their practical value was quickly and abundantly proved. Here a number are already working, and it is proposed to set them up throughout the area served by the hospital-i.e., principally Berks, Bucks, and Oxon.

In starting a clinic a suitable centre, generally a market town, is chosen, the county medical officer of health and the local doctors are consulted, so that, if possible, it may be held in the cottage hospital or in rooms which are used for infant welfare or other health purposes. Representatives of the B.R.C.S., Infant Welfare, Nursing Federation, or other voluntary associations are asked to form a local committee to run the clinic, help in its work, contribute to the expenses, and act as almoners. This committee might well become a branch of the Invalid Children's Aid Association. Sisters specially trained in orthopædic work visit the clinics, which are held every week or every fortnight, according to the need. They see the children coming from the surrounding district and patch or renew their plasters, make sure that their appliances and boots are effective, and so on. These after care sisters live at, or near, the hospital, and follow closely the treatment of any cases from their districts which are in the hospital, so that there may be no break in continuity of purpose. The sisters never initiate treatment, their work is to carry out the treatment ordered by the surgeon. The latter visits the clinics to see all the cases once every eight weeks, and makes a special additional visit when necessary. Children who are not actually sent up by their doctor are referred to him before their treatment is com menced. A list of the regular visits of the surgeon is sent to the doctors in the locality, who are thus brought into touch with the work of the hospital, and can readily refer cases for treatment or consideration. They can keep in touch with a patient right through, for they are always very welcome at the clinics or the hospital.

This coöperation is really an extension of team-work, and means much to all concerned. To the hospital it means the possibility of early treatment and thorough aftercare; to the doctors it is helpful in that, otherwise, the management of cases requiring much plaster work, all sort 
of apparatus, special boots, \&c., is difficult and troublesome; to public health representatives it is valuable, as the work concerns, mainly, children for whose treatment they are responsible."

This system has proved completely successful. Cases are now sent to the local clinics or the hospital in the early stages; this change is progressive, as the parents, doctors, school medical officers, nurses, health visitors, \&c., realise more and more fully what is being done for the various conditions and the importance of losing no time; and the after-treatment is carried on till all danger of relapse is past.

To the doctors the clinics are a great help. The cases treated need plasters and appliances often for very long periods, and these require constant care, alteration, or renewal, work practicable when one is always at it and there are hospital workshops at one's elbow, but in private practice or for a general surgeon almost impossible. Perhaps the commonest cases attending the clinics are those of past acute poliomyelitis with resultant paralyses and deformities. In a young child there are years of treatment by plaster and appliances ahead: first, the correction of deformities and the prolonged postural treatment of the muscles; then the consideration as to further measures to restore lost muscle function, or to stabilise joints. And often there is a time of waiting until the child reaches an age when an operation which is required can best be carried out. It is, for example, disadvantageous to do an arthrodesis until the subject is about 8 or 10 years old. Sir Robert Jones has said: "There is plenty to be done during the waiting, and if this be done well perhaps no operation will be needed."' Even long-neglected cases of this disease often show astonishing improvement when paralysed muscles are treated by constant relaxation for long periods with simple re-education.

All this work is difficult and tedious and hardly practicable except by means of local clinics. They are really extensions of the hospital out-patient department, and serve to bring the hospital within easy reach of the parents and the doctor. A frequent long journey with a cripple child is quite impracticable for most workingclass parents who have a house and perhaps other children to look after. Unless a clinic is available within a reasonable distance of their home they sooner or later fail to bring the child up for treatment or supervision.

Early ascertainment.-A deformity or the warning sign of a crippling disease may be noticed first by a doctor, a midwife, a school medical officer, or infantwelfare worker. It is evident (1) that all these people must appreciate the importance of immediate action (2) that ready means of getting the cases to hospital or clinic quickly must exist. It is only by the actual intercourse between hospital staff and primary health workers affected by the district-working hospital and its clinics that the necessary knowledge is attained and facilities for prompt diagnosis and treatment provided.

The problem in big cities. - In London and other big cities the problem is analogous, and can be solved in the same way. Instead of one central hospital school there might well be institutions of similar type in the country attached to orthopædic hospitals or orthopædic departments of general hospitals. Instead of a large rural district with a population a quarter or half a million, a district of the town of somewhat similar population might be assigned to each unit. The hospital would be responsible for working its district on lines which would ensure early ascertainment, continuity of treatment, and thorough after-care.

$$
\text { Finance. }
$$

Establishment of hospitals.-.Total new construction will often be avoidable. Many suitable or adaptable hospitals exist, either already devoted to work of this type, old military hospitals, or those still in use by the Ministry of Pensions. Where new building is necessary it should be of a simple type, and have the twin virtues of low establishment and low maintenance costs. Such a hospital has been described elsewhere. ${ }^{4}$ It should be of semi-permanent construction, for even so it may outlast

3 Brit. Med. Jour., Dec. 9th, 1911.
The Care of Cripple Children, Brit. Med. Jour., May 22nd, 1920. its author's generation, and eventually be considered quite out of date and ineffective. Establishment grants from the Ministry of Health are available for the pro portion of beds devoted to non-pulmonary tuberculosis.

Maintenance.-If the hospital school is " approved" by the Board of Education and by the Ministry of Health the maintenance costs can be claimed from the local authorities responsible for each case, provided they authorise the admission. The authorities can collect what is fair from the parents, and can demand from the Ministry of Health and the Board of Educa tion 50 per cent. of their own expenditure. The average weekly hospital expenses can be divided by the number of in-patient children. The sum thus arrived at can be charged to the authorities; the charges for cases coming under the education authorities must first be approved by the Board of Education.

The responsible authorities are: (1) For all tuberculous cases and for all children under school age, the local public health authority; (2) for children of school age (non-tuberculous), the local education authority.

\section{Conclusions.}

1. There are a great many cripple children in this country and a still larger number of adults whose crippledom dates from childhood.

2. Most of these cases need not have been permanently crippled if they had been given adequate treatment at an early stage of their disability.

3. Of cripple children, about one-third suffer from non-pulmonary tuberculosis, and about two-thirds from other crippling conditions.

4. The need for the adequate provision of treatment for these disabilities is very great; it has been recognised and provided for by legislation.

5. Unless a coördinated and comprehensive policy is adopted there will be a muddle-in some places a good scheme, in others a bad one, in others, again, for a long time none at all.

6. For the treatment truly to meet the need it must be $(a)$ very early, and so largely preventive; (b) efficient in suitably sited, equipped, and staffed hospitals; (c) completed by thorough after-care.

7. Very early treatment and thorough after-care can only be achieved by a hospital centred in a "district," and responsible for working that district by coöperation with other health workers.

8. Every area in the country must be served by a hospital for cripple children.

9. The special hospital conditions necessary for the various types of crippling disease, paralysis, or deformity are almost identical. A hospital satisfying the needs of the tuberculous cripple will provide all that the others require. The "local clinic" organisation is needed for all types.

10. In certain places hospitals for all varieties of cripple children already exist. Several already have a local clinic system; in others it is as yet undeveloped. In one district, where the organisation has been working for some years, the results have been most satisfactory.

11. In any district where there is a hospital for nonpulmonary tuberculous cases, and no provision for other types, that institution should enlarge its scope. To set up a separate hospital and clinic organisation for the non-tuberculous cripples would be foolish and extravagant; to leave them without provision a great deal worse.

12. The hospital which exists to deal with cases sent from a distance, with arthritis deformity or paralysis established, will become a thing of the past. Such hospitals have done magnificent work, and have been the honoured teachers of us all; but the treatment of crippling disabilities is becoming more and more of a preventive nature.

13. The establishment of this system of treatment means to substitute early and thorough treatment for what has hitherto been tardy, difficult, costly, and incomplete.

14. The expense will now be definitely associated with this treatment instead of hidden in a mass of other work, but it will be justified on economic grounds alone; many who would otherwise be sources of loss will thus become earners and producers. 